JeAn CARL SILVA ${ }^{1}$

AuGusto RADÜNZ dO AMARAL ${ }^{2}$

BRUNA DA SILVA FERREIRA ${ }^{2}$

JOÃO FRANCISCO PETRY ${ }^{2}$

MARIANA Ribeiro E SILVA²

PÂmella Caroline Krelling ${ }^{2}$

\title{
Obesidade durante a gravidez: resultados adversos da gestação e do parto
}

\author{
Obesity during pregnancy: gestational complications and birth outcomes
}

Artigo Original

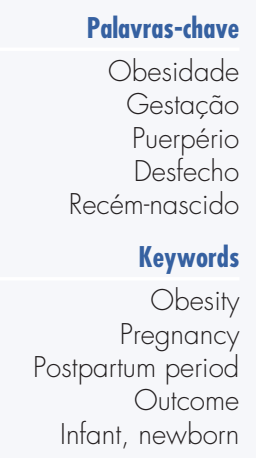

Infant, newborn
Correspondência
Jean Carl Silva
Rua Paulo Malschitzki, 10 - Zona Industrial Norte - Bom Retiro
CEP: $89219-710$
Joinville (SC), Brasil
Recebido
$12 / 05 / 2014$

Aceito com modificaçōes

$12 / 09 / 2014$

\section{Resumo}

OBJETIVO: Avaliar a influência do excesso de peso materno na gestação, no parto e nos desfechos neonatais. MÉTODOS: Estudo transversal e retrospectivo que incluiu 298 puérperas. As informações foram obtidas por meio de entrevistas e acesso aos prontuários das pacientes. As puérperas foram divididas em três grupos, conforme o índice de massa corpórea pré-gestacional: normal $\left(18,5-24,9 \mathrm{~kg} / \mathrm{m}^{2}\right)$; sobrepeso $\left(25,0-29,9 \mathrm{~kg} / \mathrm{m}^{2}\right)$ e obesidade $\left(\geq 30,0 \mathrm{~kg} / \mathrm{m}^{2}\right)$. Foram construídos modelos de regressão logística multinominal para ajustar o efeito das variáveis de confusão. Estabeleceram-se intervalos de confiança de 95\% (IC95\%). RESULTADOS: Comparadas às gestantes com peso normal, pacientes com sobrepeso apresentaram chances maiores de cesariana, sendo a odds ratio (OR) de 2,2 e IC95\% 1,3-3,9, e as obesas tiveram ainda maiores $(O R=4,2 ; 1 C 95 \% 2,1-8,1)$. As chances de desenvolvimento de diabetes gestacional aumentaram nos grupos Sobrepeso $(O R=2,5$; IC95\% 1, 1-5,6) e Obesidade (OR=1 1, 1; IC95\% 5,0-24,6). A síndrome hipertensiva na gravidez também se mostrou mais provável nas gestantes com sobrepeso $\mid \mathrm{OR}=3,2$; IC95\% 1,2-8, 1) e obesas $(\mathrm{OR}=7,5$; IC95\% 2,9-19,1). A hemorragia de grande porte no momento do parto somente apresentou maiores valores no grupo de obesas $(\mathrm{OR}=4,1$; IC95\% 1, 1-15,8). Quanto aos recém-nascidos, a probabilidade de Apgar baixo no primeiro minuto foi superior entre as obesas $(O R=5,5$; IC95\% 1,2-23,7), e a ocorrência de macrossomia aumentou nas mulheres com sobrepeso $(O R=2,9 ;$ IC95\% 1,3-6,3). Os resultados quanto à hipoglicemia neonatal não foram conclusivos. CONCLUSÃO: As chances de intercorrências maternas (diabetes gestacional, síndrome hipertensiva, hemorragia pós-parto) e neonatais (cesariana, macrossomia e escore Apgar baixo) foram maiores nos grupos com excesso de peso (sobrepeso e obesidade).

\section{Abstract}

PURPOSE: To evaluate the influence of maternal obesity on pregnancy, childbirth, and neonatal outcomes. METHODS: A cross-sectional study with 298 postpartum women. Information was obtained through interviews and access to patients' medical records. The patients were divided into three groups according to their pre-gestational body mass index: normal weight $\left(18.5-24.9 \mathrm{~kg} / \mathrm{m}^{2}\right)$, overweight $\left(25.0-29.9 \mathrm{~kg} / \mathrm{m}^{2}\right)$, and obese $\left(\geq 30.0 \mathrm{~kg} / \mathrm{m}^{2}\right)$. Data are reported as adjusted odds ratios with $95 \%$ confidence interval $(95 \% \mathrm{Cl})$ following multinomial logistic regression analysis to account for confounding variables. RESULTS: Compared to pregnant women with normal body mass index, overweight women had greater chances of having cesarean delivery, odds ratio $(\mathrm{OR})$ of 2.2 and $95 \% \mathrm{Cl}$ 1.3-3.9, and obese women even more $(O R=4.2 ; 95 \% \mathrm{Cl} 2.1-8.1)$. The chances of gestational diabetes increased in the Overweight $(O R=2.5$; $95 \% \mathrm{Cl} 1.1-5.6)$ and Obese groups $(\mathrm{OR}=11.1 ; 95 \% \mathrm{Cl} 5.0-24.6)$. The occurrence of hypertensive syndrome was also higher in overweight $(O R=3.2 ; 95 \% \mathrm{Cl} 1.2-8.1)$ and obese pregnant women $(O R=7.5 ; 95 \% \mathrm{Cl} 2.9-19.1)$. Major postpartum hemorrhage only showed greater values in the obese women group $(O R=4.1 ; 95 \% \mathrm{Cl} 1.1-15.8)$. Regarding the newborns, the probability of a low Apgar score at first minute was higher in the Obese Group (OR=5.5; $95 \% \mathrm{Cl}$ 1.2-23.7) and chances of macrosomia were higher in the Overweight Group $(O R=2.9 ; 95 \% \mathrm{Cl} 1.3-6.3)$. Data regarding neonatal hypoglycemia were not conclusive. CONCLUSION: Excessive weight (overweight and obesity) during pregnancy increases the chance of maternal complications (gestational diabetes, hypertensive syndrome, and major postpartum hemorrhage) and neonatal outcomes (cesarean delivery, macrosomia, and low Apgar score).
Serviço de Alto Risco da Maternidade Darcy Vargas - Joinville (SC), Brasil.

'Setor de Alto Risco da Maternidade Darcy Vargas, Universidade da Região de Joinville - UNIVILLE - Joinville (SC), Brasil.

${ }^{2}$ Curso de Graduação em Medicina, Universidade da Região de Joinville - UNIVILLE - Joinville (SC), Brasil.

Conflito de interesses: não há. 


\section{Introdução}

A obesidade é definida como uma síndrome metabólica crônica e multifatorial oriunda de um desequilíbrio entre alimentação e gasto calórico. É característico desse distúrbio o aumento do número e tamanho das células adiposas no organismo ${ }^{1,2}$. Segundo a Organização Mundial de Saúde (OMS) ${ }^{3}$, o número de obesos no mundo em 2005 era de 400 milhões, espera-se para 2015 um valor estimado de 700 milhões. No Brasil, o excesso de peso atingia, até 2011 , cerca de $48,5 \%$ da população, segundo levantamento da Vigilância de Fatores de Risco e Proteção para Doenças Crônicas por Inquérito Telefônico (VIGITEL) ${ }^{4}$. Quando associado à gravidez, tal problema é enfrentado em 25 a $30 \%$ das gestações no Brasil, conforme levantamentos realizados nas principais capitais brasileiras 5 .

Há uma notória dificuldade nas diretrizes sobre manejo terapêutico das gestantes de alto risco, quanto à definição de obesidade no período gravídico. Alguns autores sugerem como parâmetro o índice de massa corpórea (IMC) pré-gestacional, enquanto outros utilizam o peso corporal em relação ao ideal ${ }^{6-8}$. Embora essa dificuldade esteja com frequência presente, diversos estudos têm buscado verificar a associação entre o excesso de peso materno durante a gestação e as consequências sobre a mãe e o feto. Poucos desses estudos, entretanto, têm quantificado a influência que o excesso de peso materno exerce na gestante e no feto.

Por esse motivo, o objetivo deste estudo foi avaliar e quantificar a influência do excesso de peso materno durante a gestação, de acordo com o IMC pré-gestacional, na gestação, no parto e nos desfechos neonatais.

\section{Métodos}

Foi realizado um estudo de corte transversal com puérperas internadas na Maternidade Darcy Vargas (Joinville, Santa Catarina) entre os meses de maio e junho de 2013. Os dias de coleta foram aleatórios e sistematizados, de modo a reduzir as chances de viés de seleção. As informações foram obtidas por entrevistas e pelo acesso aos prontuários das pacientes, após leitura e assinatura do Termo de Consentimento Livre e Esclarecido (TCLE).

Foram incluídas todas as gestantes internadas na maternidade nos dias designados para coleta, com idade igual ou superior a 18 anos e que tiveram feto único. Aquelas que apresentaram dados incompletos ou com IMC inferior a $18,5 \mathrm{~kg} / \mathrm{m}^{2}$ foram excluídas.

As medidas antropométricas foram calculadas para cada mulher. O IMC $\left(\mathrm{kg} / \mathrm{m}^{2}\right)$ pré-gestacional foi classificado a partir dos critérios da OMS como de baixo peso $(<18,5)$, normal $(18,5-24,9)$, sobrepeso $(25,0-29,9)$ e obesidade $(\geq 30,0)$. Para este estudo, considerou-se hemorragia de grande porte como qualquer sangramento excessivo durante o parto que necessitou de intervenção pelo médico assistente. Foram considerados baixos escores de Apgar valores menores do que sete. Além disso, em avaliações diferentes, foram adotados dois critérios de peso para os recém-nascidos. Considerou-se macrossômicos, neonatos com peso maior que $4.000 \mathrm{~g}$, e grandes para idade gestacional (GIG), recém-nascidos situados acima do percentil 90 da curva proposta por Battaglia e Lubchenco?.

As informações recuperadas foram inseridas no software Epidata versão 3.1 e exportadas para o Microsoft Office Excel 2010. Os dados foram tratados estatisticamente, por meio do software Statistical Package for the Social Sciences (SPSS), versão 21.0. Para as variáveis quantitativas, foram calculadas as médias e os desvios padrão e, para as qualitativas, as frequências absolutas e relativas. Foram construídos modelos de regressão logística multinominal de modo a examinar a influência do excesso de peso materno nos desfechos avaliados e ajustar o efeito das variáveis de confusão. Intervalos de confiança de 95\% (IC95\%) foram estabelecidos, sendo que eram considerados valores significativos quando $\mathrm{p}<0,05$.

O projeto de pesquisa foi aprovado pelo Comitê de Ética e Pesquisa em Seres Humanos da Universidade da Região de Joinville (UNIVILLE), sob parecer 237.243. O presente estudo foi realizado de acordo com as Diretrizes e Normas Regulamentadoras de Pesquisa envolvendo seres humanos (Resolução no 466/2012 do Conselho Nacional de Saúde).

\section{Resultados}

Dentre as 327 puérperas inicialmente entrevistadas, somente 298 apresentaram os critérios necessários para este estudo. A Tabela 1 apresenta as características epidemiológicas e clínicas das pacientes. Destas, 161 $(54,1 \%)$ possuíam IMC normal, $84(28,1 \%)$ sobrepeso e $53(17,8 \%)$ obesidade. A etnia mais prevalente entre todos os grupos de IMC foi branca com $135(83,8 \%)$, $74(88,1 \%)$ e $43(81,2 \%)$, respectivamente normal, sobrepeso e obesidade. No grupo com sobrepeso, apenas oito $(9,5 \%)$ pacientes declararam-se fumantes, o maior percentual entre todos os grupos de IMC. Quanto às doenças anteriores à gestação, as pacientes obesas apresentavam com maior frequência diabetes melito tipo II $(3,7 \%)$ e hipertensão arterial sistêmica $(5,6 \%)$.

A Tabela 2 compara as chances de desfechos materno-fetais de acordo com os IMC das pacientes. Comparadas às gestantes com peso normal, pacientes com 
sobrepeso apresentaram chances maiores de cesariana, sendo a odds ratio (OR) de 2,2 e IC95\% 1,3-3,9, e as obesas tiveram ainda maiores $(\mathrm{OR}=4,2$; IC95\% $2,1-8,1)$. O desenvolvimento do diabetes melito gestacional (DMG) foi mais provável em ambas as

Tabela 1. Caracteristicas gerais da amostra (epidemiologia e antecedentes)

\begin{tabular}{|c|c|c|c|c|c|c|}
\hline \multirow{2}{*}{ Características gerais } & \multicolumn{2}{|c|}{ IMC normal } & \multicolumn{2}{|c|}{ IMC sobrepeso } & \multicolumn{2}{|c|}{ IMC obesidade } \\
\hline & n & $\%$ & n & $\%$ & n & $\%$ \\
\hline \multicolumn{7}{|l|}{ Ełnia } \\
\hline Branco & 135 & 1358 & 74 & 88,1 & 43 & 81,2 \\
\hline Preto & 7 & 4,4 & 1 & 1,2 & 5 & 9,4 \\
\hline Amarelo & 5 & 3,1 & 0 & 0,0 & 0 & 0,0 \\
\hline Pardo & 12 & 7,5 & 8 & 9,5 & 5 & 9,4 \\
\hline Indígena & 2 & 1,2 & 1 & 1,2 & 0 & 0,0 \\
\hline Total & 161 & 100 & 84 & 100 & 53 & 100 \\
\hline Fumante & 15 & 9,3 & 8 & 9,5 & 2 & 3,8 \\
\hline Idade - Média $\pm D P$ & \multicolumn{2}{|c|}{$27,0 \pm 6,5$} & \multicolumn{2}{|c|}{$29,8 \pm 6,5$} & \multicolumn{2}{|c|}{$29,42 \pm 6,3$} \\
\hline \multicolumn{7}{|l|}{ Gesta } \\
\hline Gesta 1 & 56 & 568 & 20 & 23,8 & 16 & 30,2 \\
\hline Gesta 2 & 54 & 545 & 23 & 27,4 & 22 & 41,5 \\
\hline Gesta 3+ & 51 & 517 & 41 & 48,8 & 15 & 28,3 \\
\hline Total & 161 & 100 & 84 & 100 & 53 & 100 \\
\hline \multicolumn{7}{|l|}{ Condições anteriores à gestação } \\
\hline Diabetes melito tipo II & 2 & 1,2 & 0 & 0,0 & 2 & 3,7 \\
\hline Hipertensão arterial sistêmicu & 2 & 1,2 & 3 & 3,5 & 3 & 5,6 \\
\hline
\end{tabular}

IMC: índice de massa corpórea; DP: desvio padrão; Gesta: número de gestações.

Tabela 2. Desfechos materno-fetais; comparacãao entre os grupos de acordo com o índice de massa corpórea

\begin{tabular}{|c|c|c|c|c|}
\hline \multirow{2}{*}{ Desfechos materno-fetais } & \multirow{2}{*}{$\begin{array}{c}\text { Grupo IMC } \\
\left(\mathrm{kg} / \mathrm{m}^{2}\right)\end{array}$} & \multicolumn{2}{|c|}{$\begin{array}{c}\text { Valores absolutos } \\
\text { e relativos }\end{array}$} & \multirow{2}{*}{$\begin{array}{l}\text { Odds ratio } \\
\text { (IC95\%) }\end{array}$} \\
\hline & & n & $(\%)$ & \\
\hline \multirow{3}{*}{ Pré-termo } & $18,5-24,9$ & 12 & 7,4 & - \\
\hline & $25,0-29,9$ & 3 & 3,5 & $0,4(0,1-1,6)$ \\
\hline & $\geq 30,0$ & 2 & 3,7 & $0,5(0,1-2,2)$ \\
\hline \multirow{3}{*}{ Cesariana } & $18,5-24,9$ & 43 & 26,7 & - \\
\hline & $25,0-29,9$ & 38 & 45,2 & $2,2(1,3-3,9)$ \\
\hline & $\geq 30,0$ & 32 & 60,4 & $4,2(2,1-8,1)$ \\
\hline \multirow{3}{*}{ DMG } & $18,5-24,9$ & 12 & 7,4 & - \\
\hline & $25,0-29,9$ & 14 & 16,6 & $2,5(1,1-5,6)$ \\
\hline & $\geq 30,0$ & 25 & 47,1 & $11,1(5,0-24,6$ \\
\hline \multirow{3}{*}{ DHEG } & $18,5-24,9$ & 8 & 4,9 & - \\
\hline & $25,0-29,9$ & 12 & 14,3 & $3,2(1,2-8,1)$ \\
\hline & $\geq 30,0$ & 15 & 28,3 & $7,5(2,9-19,1)$ \\
\hline \multirow{3}{*}{ HGP } & $18,5-24,9$ & 4 & 2,5 & - \\
\hline & $25,0-29,9$ & 6 & 7,1 & $3,0(0,8-11,0)$ \\
\hline & $\geq 30,0$ & 5 & 9,4 & $4,1(1,1-15,8)$ \\
\hline
\end{tabular}

IMC: índice de massa corpórea; IC95\%: intervalo de confiança de 95\%; DMG: diabetes melito gestacional; DHEG: doença hipertensiva específica da gravidez; HGP: hemorragia de grande porte no momento do parto. categorias com excesso de peso, nos grupos Sobrepeso $(\mathrm{OR}=2,5$; IC95\% 1,1-5,6) e Obesidade $(\mathrm{OR}=11,1$; IC95\% 5,0-24,6). A ocorrência de doença hipertensiva específica da gravidez (DHEG) foi mais prevalente nas gestantes com sobrepeso $(\mathrm{OR}=3,2$; IC95\% 1,2-8,1) e obesas $(\mathrm{OR}=7,5$; IC95\% 2,9-19,1).

No momento do parto, a ocorrência de hemorragia de grande porte somente apresentou valores superiores no grupo de obesas (OR =4,1; IC95\% 1,1-15,8), sendo, portanto, neste estudo, característica exclusiva de gestantes com altos IMC. As comparações quanto às chances de ocorrência de recém-nascidos prematuros não foram significativas.

A Tabela 3 apresenta a razão de chances dos desfechos perinatais, conforme a categoria do IMC de cada paciente. A probabilidade de Apgar baixo no primeiro minuto foi mais elevada entre os recém-nascidos de gestantes obesas (OR =5,5; IC95\% 1,2-23,7), mas não se mostrou significativa quando comparada ao grupo com sobrepeso. Entretanto, a ocorrência de recém-nascidos GIG aumentou entre as mulheres com sobrepeso $(\mathrm{OR}=2,9$; IC95\% 1,3-6,3) e não foi significativa no grupo com obesidade. À medida que o grupo de IMC variava de normal à obesidade, o índice de macrossomia aumentou gradativamente. Os resultados quanto à hipoglicemia neonatal, às doenças cardiorrespiratórias e à internação em Unidade de Terapia Intensiva (UTI) não foram conclusivos.

Tabela 3. Desfechos perinatais; comparação entre os grupos de acordo com o índice de massa corpórea

\begin{tabular}{|c|c|c|c|c|}
\hline \multirow[t]{2}{*}{ Desfechos perinatais } & \multirow{2}{*}{$\begin{array}{c}\text { Grupo IMC } \\
\left(\mathrm{kg} / \mathrm{m}^{2}\right)\end{array}$} & \multicolumn{2}{|c|}{$\begin{array}{c}\text { Valores absolutos } \\
\text { e relativos }\end{array}$} & \multirow{2}{*}{$\begin{array}{l}\text { Odds ratio } \\
\text { (IC95\%) }\end{array}$} \\
\hline & & $n$ & $(\%)$ & \\
\hline \multirow{3}{*}{ Apgar-1 baixo } & $18,5-24,9$ & 3 & 1,8 & - \\
\hline & $25,0-29,9$ & 3 & 3,5 & $1,9(0,4-9,8)$ \\
\hline & $\geq 30,0$ & 5 & 9,4 & $5,5(1,2-23,7)$ \\
\hline \multirow{3}{*}{ PIG } & $18,5-24,9$ & 9 & 5,6 & - \\
\hline & $25,0-29,9$ & 3 & 3,5 & $0,6(0,1-2,3)$ \\
\hline & $\geq 30,0$ & 5 & 9,4 & $1,7(0,5-5,5)$ \\
\hline \multirow{3}{*}{ AIG } & $18,5-24,9$ & 139 & 86,3 & - \\
\hline & $25,0-29,9$ & 64 & 76,1 & $0,5(0,2-0,9)$ \\
\hline & $\geq 30,0$ & 40 & 75,4 & $0,5(0,2-1,1)$ \\
\hline \multirow{3}{*}{ GIG } & $18,5-24,9$ & 13 & 8,1 & - \\
\hline & $25,0-29,9$ & 17 & 20,2 & $2,9(1,3-6,3)$ \\
\hline & $\geq 30,0$ & 8 & 15,1 & $2,0(0,8-5,2)$ \\
\hline \multirow{3}{*}{ Hipoglicemia neonatal } & $18,5-24,9$ & 4 & 2,5 & - \\
\hline & $25,0-29,9$ & 3 & 3,6 & $1,4(0,3-6,6)$ \\
\hline & $\geq 30,0$ & 2 & 3,7 & $1,5(0,2-8,6)$ \\
\hline
\end{tabular}

IMC: índice de massa corpórea; IC95\%: intervalo de confiança de 95\%; Apgar-1 baixo: escore Apgar ao primeiro minuto <7; PIG: pequeno para idade gestacional

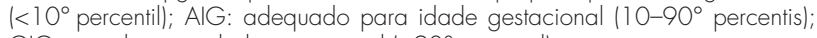
GIG: grande para idade gestacional $\left(>90^{\circ}\right.$ percentil). 


\section{Discussão}

Este estudo mostrou o impacto da dicotomia obesidade e gestação sobre resultados gestacionais e perinatais. Além disso, por meio dos resultados obtidos, foi possível quantificar o risco da obesidade materna para cada desfecho individualmente, permitindo ao leitor a avaliação dos diferentes graus de influência que o excesso de peso materno exerceu em cada variável analisada.

Dentre os resultados encontrados, observou-se que a etnia mais prevalente entre todos os grupos de IMC foi branca. Essa característica se assemelha aos resultados de Sebire et al. ${ }^{10}$, no qual, em todas as categorias de IMC, a etnia branca obteve percentuais superiores. Uma razão para tal semelhança é a composição da amostra do nosso estudo, visto que a região Sul do país e, em especial a população do estado de Santa Catarina, teve uma forte colonização europeia ${ }^{11}$.

Além disso, era esperado que o grupo de obesas apresentasse maior média de idade. No entanto, os presentes resultados indicaram maior média entre as puérperas com sobrepeso, contrariando a associação positiva entre obesidade e idade avançada presente em outros estudos ${ }^{10-13}$. Também havia a expectativa de que no grupo de obesas houvesse maior frequência de doenças crônicas anteriores à gestação e, nesse caso, isso foi confirmado tanto nesse estudo como em outros ${ }^{10,14}$.

Aliyu et al. ${ }^{15}$ apresentaram em seu estudo a associação entre obesidade materna e ocorrência de parto instrumental. Adamo et al. ${ }^{16}$ também verificaram um número maior de cesarianas em pacientes com IMC superiores aos valores normais. Esses resultados convergem com os encontrados neste estudo, no qual a chance de parto normal foi reduzida em cinco vezes nas mulheres obesas.

A ocorrência de diabetes gestacional e DHEG tem sido associada diretamente ao excesso de peso da gestante $^{10,13,17,18}$. Com relação ao diabetes, o aumento do número e tamanho dos adipócitos promove um agravamento da resistência à insulina, que, associado ao hormônio lactogênio placentário, favorece o desenvolvimento do $\mathrm{DMG}^{19}$.

Em nosso estudo, a relação entre obesidade materna e prematuridade não se mostrou conclusiva. Quando acessada a literatura, os estudos apresentavam dados divergentes. Houve trabalhos em que o excesso de peso reduziu as chances de prematuridade ${ }^{10} \mathrm{e}$ outros em que houve o aumento da ocorrência de pré-termos ${ }^{13}$.

Quanto aos desfechos perinatais, o excesso de peso em nossa amostra aumentou as chances de escore Apgar baixo ao primeiro minuto e ocorrência de recém-nascidos GIG, conforme outras publicações que identificaram as mesmas associações ${ }^{20}$. Não foi possível, neste estudo, associar a obesidade materna à hipoglicemia neonatal, à ocorrência de doenças cardiovasculares e à internação em UTI neonatal, uma vez que a razão entre as probabilidades não foi significativa. Outros autores, entretanto, identificaram uma maior frequência desses desfechos em gestantes com IMC elevado ${ }^{10,21,22}$.

Em conclusão, observamos que os grupos com maiores IMC (sobrepeso e obesidade) apresentaram maiores chances de complicações no parto (cesariana e hemorragia de grande porte) e intercorrências maternas (diabetes gestacional e síndrome hipertensiva). Também mostraram maiores chances de intercorrências perinatais (escore Apgar baixo ao primeiro minuto e macrossomia). Entre os grupos, as mulheres obesas tiveram chances mais elevadas de complicações.

\section{Referências}

1. Oliveros E, Somers VK, Sochor O, Goel K, Lopez-Jimenez F. The concept of normal weight obesity. Prog Cardiovasc Dis. 2014;56(4):426-33.

2. Pi-Sunyer FX. The obesity epidemic: pathophysiology and consequences of obesity. Obes Res. 2002;10(Suppl 2):97S-104S.

3. World Health Organization. Obesity: preventing and managing the global epidemic. WHO Technical Report Series 894. Geneva: WHO; 2000.

4. Brasil. Ministério da Saúde [Internet]. Vigitel Brasil 2011 : Vigilância de fatores de risco e proteção para doenças crônicas por inquérito telefônico. Brasília (DF): Ministerio da Saúde; 2012 [citado 2014 Jan 10]. Disponível em: http://www.dive.sc.gov.br/conteudos/ agravos/Dant/VIGITEL-2011.pdf
5. Paiva LV, Nomura RMY, Dias MCG, Zugaib M. Obesidade materna em gestações de alto risco e complicações infecciosas no puerpério. Rev Assoc Med Bras. 2012;58(4):453-8.

6. Isaacs JD, Magann EF, Martin RW, Chauhan SP, Morrison JC. Obstetric challenges of massive obesity complicating pregnancy. J Perinatol. 1994; 14(1): 10-4.

7. Yu CK, Teoh TG, Robinson S. Obesity in pregnancy. BJOG. 2006; 113(10):1117-25.

8. Ellwood D. What can be done about maternal obesity? Aust N Z J Obstet Gynaecol. 2008;48(3):227.

9. Battaglia FC, Lubchenco LO. A practical classification of newborn infants by weight and gestational age. J Pediatr. 1967;71 (2):159-63. 
10. Sebire NJ, Jolly M, Harris JP, Wadsworth J, Joffe M, Beard RW, et al. Maternal obesity and pregnancy outcome: a study of 287,213 pregnancies in London. Int J Obes Relat Metab Disord. $2001 ; 25(8): 1175-82$.

11. Seyferth G. A dimensão cultural da imigração. Rev Bras Cienc Soc. $2011 ; 26(77): 47-62$.

12. Seabra G, Padilha PC, Queiroz JA, Saunders C. [Pregestational overweight and obesity: prevalence and outcome associated with pregnancy]. Rev Bras Ginecol Obstet. 2011 ;33(11):348-53. Portuguese.

13. Li N, Liu E, Guo J, Pan L, Li B, Wang P, et al. Maternal prepregnancy body mass index and gestational weight gain on pregnancy outcomes. PLoS One. 2013;8(12):e82310.

14. Baron CM, Girling LG, Mathieson AL, Menticoglou SM, Seshia MM, Cheang MS, et al. Obstetrical and neonatal outcomes in obese parturients. J Matern Fetal Neonatal Med. 2010;23(8):906-13.

15. Aliyu MH, Luke S, Wilson RE, Saidu R, Alio AP, Salihu HM, et al. Obesity in older mothers, gestational weight gain, and risk estimates for preterm phenotypes. Maturitas. 2010;66(1):88-93.

16. Adamo KB, Ferraro ZM, Goldfield G, Keely E, Stacey D, Hadiiyannakis $S$, et al. The Maternal Obesity Management (MOM) Trial Protocol: a lifestyle intervention during pregnancy to minimize downstream obesity. Contemp Clin Trials. 2013;35(1):87-96.

17. Correa PJ, Vargas JF, Sen S, Illanes SE. Prediction of gestational diabetes early in pregnancy: targeting the long-term complications. Gynecol Obstet Invest. 2014;77(3): 145-9.

18. Sarwer DB, Allison KC, Gibbons LM, Markowitz JT, Nelson DB. Pregnancy and obesity: a review and agenda for future research. J Womens Health (Larchmt). 2006; 15(6):720-33.

19. Gupta A. Prevalence of gestational Diabetes Mellitus and associated risk factors in pregnant women. N Indian J Surg. 2011 ;2(4):206.

20. Silva JC, Bertini AM, Ribeiro TE, Carvalho LS, Melo MM, Barreto Neto $L$. [Factors related to the presence of large for gestational age newborns in pregnant women with gestational diabetes mellitus]. Rev Bras Ginecol Obstet. 2009;31 (1):5-9. Portuguese.

21. Watkins ML, Rasmussen SA, Honein MA, Botto LD, Moore CA. Maternal obesity and risk for birth defects. Pediatrics. 2003;111(5 Pt 2): 1152-8.

22. Waller DK, Shaw GM, Rasmussen SA, Hobbs CA, Canfield MA, Siega-Riz AM, et al. Prepregnancy obesity as a risk factor for structural birth defects. Arch Pediatr Adolesc Med. 2007; $161(8): 745-50$. 